American Chemical Society, J. Am. Chem. Soc., ja0634831, Supporting Information Page S1

\title{
Carbons with Extremely Large Volume of Uniform Mesopores Synthesized by Carbonization of Phenolic Resin Film Formed on Colloidal Silica Template
}

\author{
Kamil P. Gierszal and Mietek Jaroniec \\ Department of Chemistry, Kent State University, Kent, Ohio 44242 USA
}

\section{Experimental}

The mesoporous carbon studied was prepared by hard templating method. The silica template used was obtained by centrifugation of commercial $\sim 24 \mathrm{~nm}$ colloidal silica (Ludox AS-40 provided by the W. R. Grace \& Co. - Davison). Ludox AS-40 is an aqueous colloidal dispersion containing $40 \%$ of silica and stabilized with ammonium hydroxide at $\mathrm{pH}$ about 9-9.5; after drying its BET specific surface area is in the range 129-155 $\mathrm{m}^{2} / \mathrm{g}$ (manufacturer's product information).

The synthesis of carbon was performed by impregnating $1 \mathrm{~g}$ of the colloidal silica template with $40 \mathrm{mg}$ of anhydrous $98 \%$-oxalic acid (used as a catalyst; purchased from Acros Organics and used as received). The impregnated silica template was filled with $0.21 \mathrm{~g}$ of $98 \%$-resorcinol (purchased from Acros Organics and used as received ) and $99 \%$-crotonaldehyde (2-butenal; purchased from Acros Organics and used as received) mixture (1:1.2 molar ratio) using an incipient wetness method. Subsequently, the composite sample in an open container was subjected to a series of thermal treatments. The first treatment was at temperature of $60{ }^{\circ} \mathrm{C}$ for $0.5 \mathrm{~h}$. The second treatment carried out at temperature of $120^{\circ} \mathrm{C}$ for $\sim 10 \mathrm{~h}$ was used to perform an initial pre-polymerization (the composite sample was of yellow-orange color). The actual polymerization was performed at $200{ }^{\circ} \mathrm{C}$ for $5 \mathrm{~h}$ (the composite sample was of soft brown color). At the last thermal treatment, the resulting sample was transferred into a tube furnace for carbonization under nitrogen at $900{ }^{\circ} \mathrm{C}$ for $2 \mathrm{~h}$ using heating rate of $2{ }^{\circ} \mathrm{C} / \mathrm{min}$. The silica template was removed below 1\% (confirmed by thermogravimetric analysis) by using $\sim 15 \%$-HF acid. The resulting carbon was rinsed with butanol (Acros Organics) and hexane (Acros Organics) and dried in a vacuum oven at $80{ }^{\circ} \mathrm{C}$ for $\sim 10 \mathrm{~h}$. The yield of this synthesis was $\sim 80 \mathrm{mg}$ of carbon per $1 \mathrm{~g}$ of colloidal silica. 
American Chemical Society, J. Am. Chem. Soc., ja0634831, Supporting Information Page S2

\section{Derivation of the relation between the pore volume and carbon film thickness}

\section{Notation:}

$\mathrm{V}_{\mathrm{s}}\left[\mathrm{cm}^{3} / \mathrm{g}\right]$ - pore volume of porous silica per gram of the silica sample $\mathrm{S}_{\mathrm{s}}\left[\mathrm{m}^{2} / \mathrm{g}\right] \quad$ - specific surface area of porous silica per gram of the sample $\mathrm{S}_{\mathrm{s}}\left[\mathrm{m}^{2} / \mathrm{cm}^{3}\right]$ - specific surface area of porous silica per $\mathrm{cm}^{3}$ of the sample (including pore volume and volume of silica; without interparticle space)

$\mathrm{d}_{\mathrm{s}}\left[\mathrm{g} / \mathrm{cm}^{3}\right] \quad-$ absolute density of amorphous silica $\left(2.2 \mathrm{~g} / \mathrm{cm}^{3}\right)$

$\mathrm{V}_{\mathrm{c}}\left[\mathrm{cm}^{3} / \mathrm{g}\right]$ - pore volume of carbon replica per gram of the carbon sample $\mathrm{V}_{\mathrm{c}}^{\prime}\left[\mathrm{cm}^{3} / \mathrm{cm}^{3}\right]$ - volume of carbon replica per $\mathrm{cm}^{3}$ of the sample (including pore volume and volume of carbon; without interparticle space)

$\mathrm{m}_{\mathrm{c}}\left[\mathrm{g} / \mathrm{cm}^{3}\right] \quad$ - envelope density of carbon replica - a mass of carbon per $\mathrm{cm}^{3}$ of the sample (including pore volume and volume of carbon; without interparticle space)

$\mathrm{t}_{\mathrm{c}}[\mathrm{m}]-$ - carbon film thickness formed on the template surface

$\mathrm{d}_{\mathrm{c}}\left[\mathrm{g} / \mathrm{cm}^{3}\right] \quad-$ absolute density of amorphous carbon $\left(2.1 \mathrm{~g} / \mathrm{cm}^{3}\right)$

$$
\begin{array}{ll}
S_{s}^{\prime}=\frac{S_{s}}{V_{s}+\frac{1}{d_{s}}} ; & V_{c}^{\prime}=\frac{S_{s}}{V_{s}+\frac{1}{d_{s}}} \cdot t_{c} \\
m_{c}=V_{c}^{\prime} \cdot d_{c} ; & m_{c}=\frac{S_{s}}{V_{s}+\frac{1}{d_{s}}} \cdot d_{c} \cdot t_{c}
\end{array}
$$

$$
V_{c}=\frac{1-V_{c}^{\prime}}{m_{c}}=\frac{1-\frac{S_{s}}{V_{s}+\frac{1}{d_{s}}} \cdot t_{c}}{\frac{S_{s}}{V_{s}+\frac{1}{d_{s}}} \cdot t_{c} \cdot d_{c}}=\frac{V_{s}+\frac{1}{d_{s}}-S_{s} \cdot t_{c}}{S_{s} \cdot t_{c} \cdot d_{c}}
$$


American Chemical Society, J. Am. Chem. Soc., ja0634831, Supporting Information Page S3

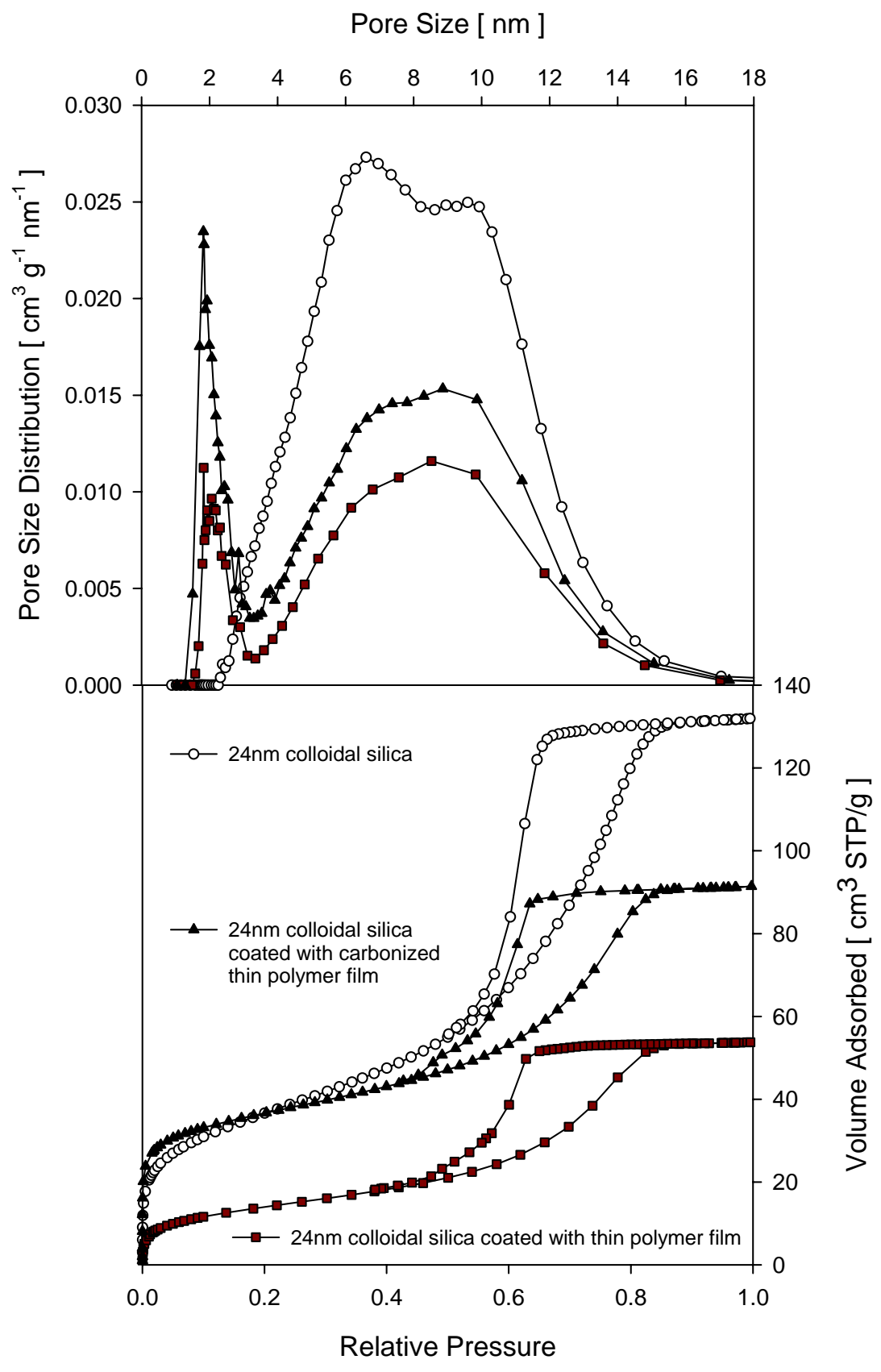

Figure 1S. Nitrogen adsorption isotherms at $-196{ }^{\circ} \mathrm{C}$ and the corresponding pore size distributions for $24 \mathrm{~nm}$ colloidal silica template (circles), $24 \mathrm{~nm}$ colloidal silica coated with a thin layer of poly(crotonaldehyde-resorcinol) (triangles), and $24 \mathrm{~nm}$ colloidal silica coated with a thin layer of carbon obtained after carbonization of poly(crotonaldehyde-resorcinol) (squares). Nitrogen adsorption isotherms were measured using a Micrometrics ASAP 2010 volumetric adsorption analyzer (Norcross, GA). Before the measurements, samples were degassed at $413 \mathrm{~K}$ for $2 \mathrm{~h}$. 
American Chemical Society, J. Am. Chem. Soc., ja0634831, Supporting Information Page S4

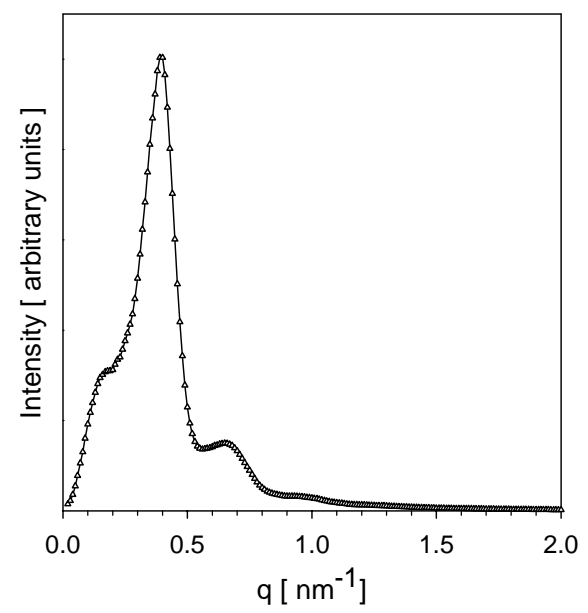

Figure 2S. Small angle X-ray scattering (SAXS) spectra for $24 \mathrm{~nm}$ colloidal silica template. The SAXS measurements were conducted at room temperature using the NanoSTAR system (Bruker AXS) with pinhole collimation and a two-dimensional detector (HiSTAR), mounted on a micro focus X-ray tube with copper anode and equipped with crossed Gobel mirrors. The resulting SAXS scans were subjected to background correction

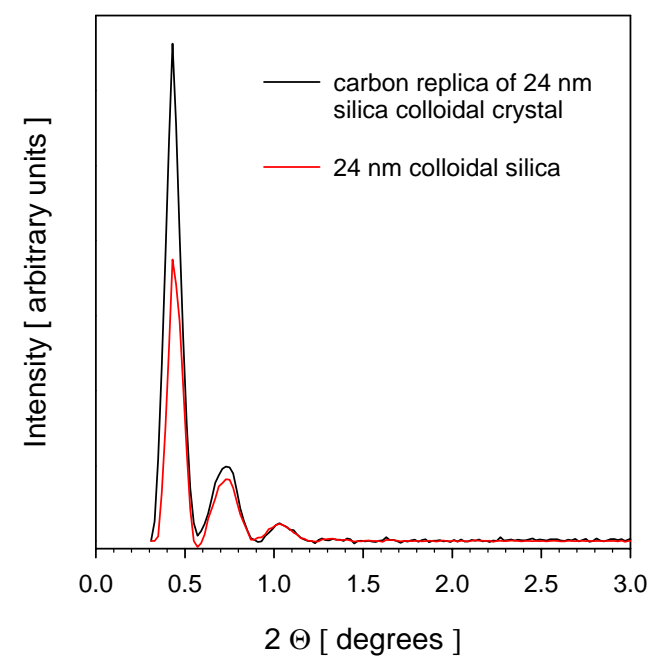

Figure 3S. Small angle X-ray diffraction (XRD) spectrum for $24 \mathrm{~nm}$ colloidal silica template and its carbon replica. Room temperature powder X-ray diffraction (XRD) data were collected on PANalytical X'Pert Pro MPD - Multi Purpose Diffractometer using CuK radiation and proportional detector. Programmable divergence slits and programmable anti-scatter slits were employed to achieve performance at the lowest angles. The multi-purpose sample stage (MPSS) was used to scan the samples in $0.02^{\circ} 2 \theta$ steps with a counting time of $20 \mathrm{~s} / \mathrm{step}$. The resulting $\mathrm{XRD}$ scans were subjected to background correction. 
American Chemical Society, J. Am. Chem. Soc., ja0634831, Supporting Information Page S5

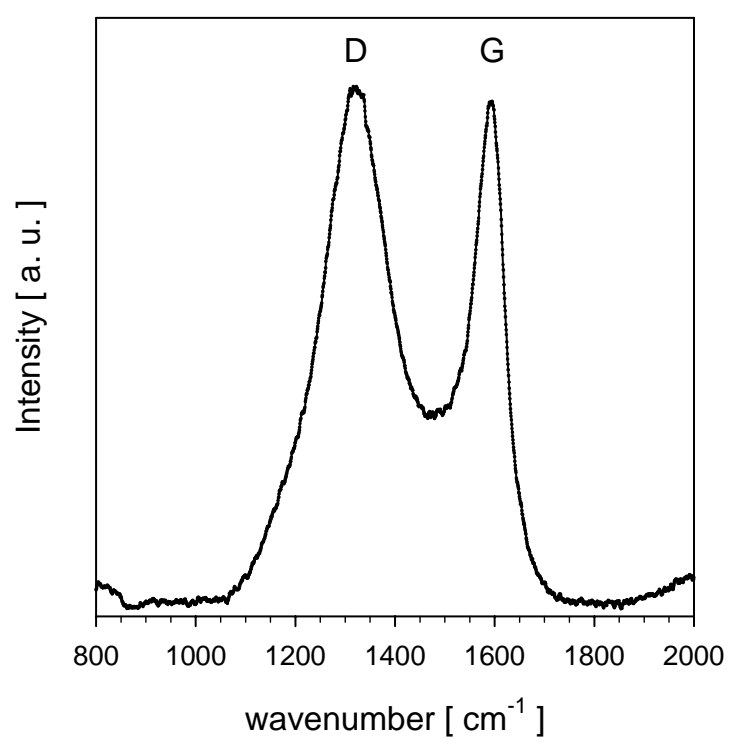

Figure 4S. Raman spectrum for the carbon replica of $24 \mathrm{~nm}$ colloidal silica template, the shape of which is typical for amorphous carbons (e.g., see Spectrochim. Acta A, 59, 2003, 2267). This spectrum was recorded on a Horiba Jobin Yvon 'LabRam' dispersion Raman microscope equipped with a CCD detector using a He-Ne laser (632 nm) and $20 \mathrm{~s}$ acquisition times.

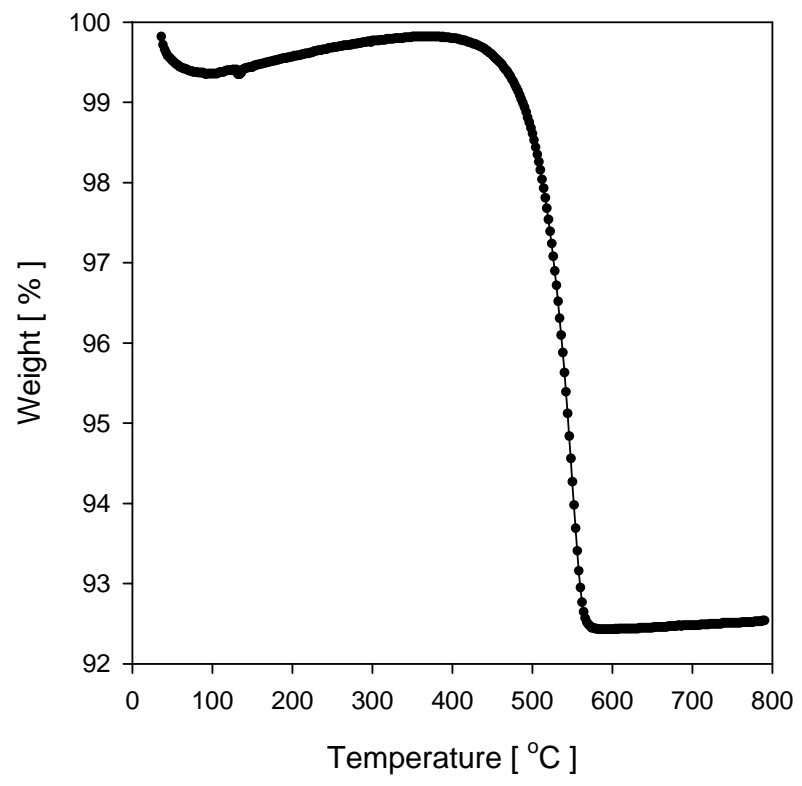

Figure 5S. Thermogravimetric (TG) data for the silica-carbon composite consisting of $24 \mathrm{~nm}$ colloidal silica template with carbon film coated on its surface. The TG analysis was carried out in flowing air using a high-resolution TGA 2950 thermogravimetric analyzer (TA Instruments, Inc., New Castle, DE); the maximum heating rate was $2{ }^{\circ} \mathrm{C} \mathrm{min}^{-1}$. 